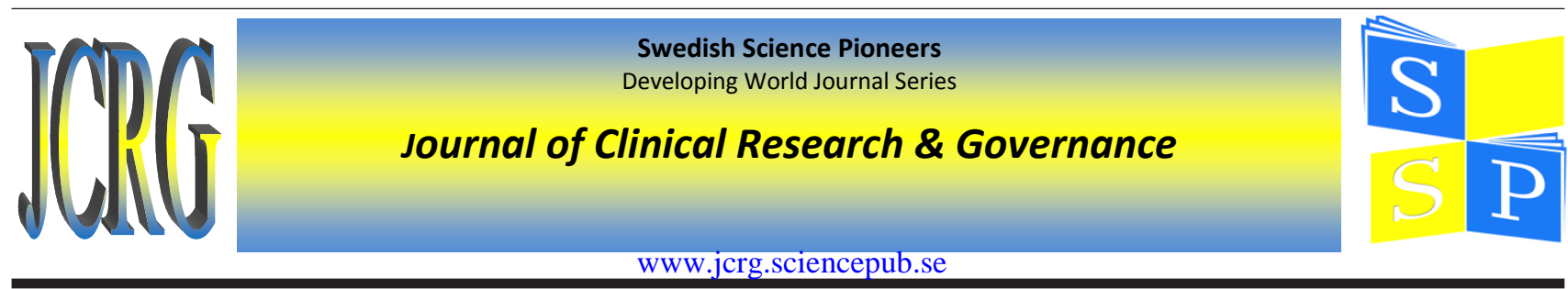

Research Article

\title{
Reliability and Validity of the Persian Version of Global School-based Student Health Survey Adapted for Iranian School Students
}

Reza Ziaei $^{\text {a }}$, Saeed Dastgiri ${ }^{\text {b }}$, Joaquim Soares ${ }^{a}$, Elaheh Baybordi ${ }^{\text {b }}$, AliHossein Zeinalzade ${ }^{\text {b }}$, Vahab Asl Rahimi ${ }^{\text {c }}$, Reza Mohammadi ${ }^{\text {d }}$

a: Department of Health Sciences, Unit for Public Health Science, Mid Sweden University, Sundsvall, Sweden.

b: Department of Community Medicine, Tabriz University of Medical Sciences, Tabriz, Iran.

c: Unit for Adolescence Health, Province Health Center, Tabriz, Iran.

d: Department of Public Health Science, Unit for Social Medicine, Karolinska Institute, Stockholm, Sweden.

Correspondence

Reza Ziaei, Department of Health Sciences, Unit for Public Health Science, Mid Sweden University, M Humlegården, 85170 Sundsvall, Sweden.

Tel: +46 723336222; Fax: +468307351.

Email: Reza.Ziaei@miun.se

\section{Keywords:}

Global School-based Student Health Survey Global Youth Tobacco Survey

Reliability

Validity

Persian

Smoking

Received: 2014-06-23

Accepted: 2014-08-25

DOI: $10.13183 /$ jcrg.v3i2.138

\section{Abstract}

Purpose: Global School-based Student Health Survey (GSHS) is a project supported by the World Health Organization to assess adolescent health-risk behaviours and protective factors. The present study reports test-retest and internal consistency reliability as well as Content Validity Index (CVI) for the Persian version of GSHS and Global Youth Tobacco Survey (GYTS) adapted for Iranian students.

Methods: The original English GSHS, GYTS and Optional Shisha modules were translated and back-translated, pilottested, reformed and corrected for clarity. The back-translated and English versions were compared and the Persian version was then edited to reach consistency for two versions. In order to evaluate the performance of this measure for our study sample $(n=47)$, we examined its test-retest reliability, internal consistency, and correlation between total test and re-test scales score as well as CVI.

Results: The internal consistency (Cronbach's alpha) for the whole GSHS Core modules was 0.69, and for CoreExpanded GSHS, GYTS and Optional Modules for Shisha was 0.90. Test-retest reliability varied between 0.51 and 1.00 for Core and 0.23 to 1.00 for Core-Expanded GYTS and Optional Modules of Shisha. Total test and re-test scales score for GSHS Core varied from 0.64 to 1.00, and for Core-Expanded GSHS, GYTS and Optional Modules of Shisha varied 0.67 to 0.91 .

Conclusion: The Persian version of GSHS Core, Core-Expanded and GYTS has acceptable reliability and validity, and can serve as a reliable instrument for Persian-speaking communities.

\section{Introduction}

Each year globally an estimated 250,000 homicide cases occur among youths of 10-29 years of age [1]. Health risk behaviors are the major leading cause of death among young people in every region of the world [2, 3]. Addressing health risk behaviors as a public health issue needs implementing of a global strategy, therefore building the foundation is essential to control and prevention of these issues. The availability of reliable data will enable experts to examine the impact of health-risk behaviors on adolescents worldwide and developing health policies and interventions to decrease undesired health impacts on population health [4]. The World Health Organization (WHO) designed and supports Global Schoolbased Student Health Survey (GSHS) to measure and assesses the behavioral risk factors and protective factors in adolescents across diverse populations. GSHS is an inexpensive school based survey which gathers data on student's health risk behavior and protective factors related to the leading causes of death across world.

GSHS is a survey which targeted students aged 13-17 years to assess their protective and risky behaviours. GSHS uses a selfreport questionnaire whose items are categorized in 10 leading cause of morbidity and mortality among students including , 
Alcohol use, Dietary behaviors, Drug use, Hygiene, Mental health, Physical activity, Protective factors, Sexual behaviors, Tobacco use as well as Violence and unintentional injury [5].

Countries can supplement questionnaires with their nationalspecific related questions to promote its flexibility. Additionally some questions suggest treating with country-specific options.

GSHS uses a standardized scientific process among participating countries to produce specific data which may be locally and internationally used to identify and respond to health priorities and to evaluate youth safety and health promotion [5].

Until present 109 countries in the world have implemented GSHS. This has resulted in 80 fact sheets, 59 datasets, and 26 full reports, posted on the WHO website [5].

The authors found just one study published on the reliability of GSHS for Fijian girls [3] and another study for the reliability of Chinese version of Global Youth Tobacco survey (GYTS) [6]. Although many countries have implemented GSHS, nothing more than one published report on the reliability of GSHS was found. In Iran GYTS questionnaire 2 times have been used for assessing the tobacco use among students in 2003 and 2007 but no reliability and validity reported for Persian Version of these questionnaires [7]. In Iran due to the lack of comprehensive and reliable instrument for collecting data on health issues in schools the present pilot study, aimed to assess the reliability and validity of the Persian version of GSHS and GYTS questionnaires.

\section{Methods}

\section{Study site and study population}

Iran is a country located in the Middle East with a population of 75,000000 . Tabriz, in which the present study was done, is situated in North West of Iran with 2,000,000 inhabitants. Persian is the formal language of instruction. There is homogeneity in the population for ethnicity and language. This city has 53,057 School students at high school stage, consisting of 29,935 female and 32,779 male students, during 2011-12 academic years. Schools are separated for girls and boys. The present pilot study is the first part of a GSHS and GYTS survey in Tabriz.

\section{Sample selection}

Inclusion criteria for participation in this study was being a high school student aged 15-17. Sampling for the study has done with the assistance of the Tabriz Medical University Epidemiology Center. 7 schools have selected from Tabriz metropolis with overall homogeneity in culture and language. All participants met eligibility requirements for school attendance, grade, and age.

All identified 7 schools by this center participated, and 58 volunteer and eligible students registered for the study. Ethical approvals for the study were obtained from the Ethics Committee of Tabriz University of Medical Sciences. Informed consent parental forms were obtained for each student. One week before the study, parents were informed about it by the school authorities. Consent forms were obtained in person from all participants parents. Consent documents were available in Persian language.

All the participants, who had been administered a self-report assessment in the first scheduled assessment visit in their schools (Time 1), participated in the re-test study (Time 2). A further eligibility criterion for the inclusion of participants in this study was their presence in school while the retest survey visit was being administered, about two weeks later (in this paper referred to as Time 2). Out of 58 participants, 11 were excluded from the study (during test and re-test) due to incomplete answer sheets or leaving the session. The response rate was $81 \%$ for the whole study and $87 \%$ for the test and $94 \%$ for the re-test participation.

Translation of Global School-based Student Health Survey
(GSHS) and Global Youth Tobacco Survey (GYTS) plus Shisha Modules

In the present study, a 335-item English version of GSHS, GYTS, plus Shisha module were used by the public health specialists for measuring reliability and validity of the Persian version questionnaires. This instrument was adapted from Core, Core-Expanded, as well as GYTS and optional items for shisha smoking which are all available on the WHO, GSHS website [5]. The items addressed: alcohol use, narcotic-drug use, tobacco use, hygiene, dietary behaviors, sexual behaviors, mental health, physical activity, violence and unintentional injury, as well as protective factors.

In order to add country-specific questions based on the GSHS guide, items were modified to be relevant to the local cultural background of Iran. All modules and items of GSHS were included in this study, except those items would not apply to Iran; for example, selling alcoholic beverages to children was excluded, because alcohol consumption is forbidden for all in Iran with no places for selling alcoholic drinks with the exception of the black market. Additionally, in Iran there are no coeducational schools, eliminating the need for items in the hygiene module that ask about separate toilets for girls and boys at schools.

Both GSHS and GYTS plus Shisha Modules questionnaires were translated from English into the Persian language by a native English-Persian speaker, and then tested with 10 adolescents as a pilot, edited and reformed for clarity, and then back-translated into English by a bilingual researcher of Persian language. The original and back-translated questionnaires were compared and the Persian version was then corrected to reach consistency in both versions. The translated Persian version was checked again by a native speaker for colloquial and grammatical correctness, resulting in the final questionnaire.

Table 1. Demographic characteristics of students in test and re-test.

\begin{tabular}{|lccc|}
\hline Age & N & $\begin{array}{c}\text { Percentage } \\
(\%)\end{array}$ \\
15 & & 8 & 17 \\
$16-17$ & & 39 & 83 \\
Total & & 47 & 100 \\
Gender & Male & 23 & 48.9 \\
& Female & 24 & 51.1 \\
Total & & 47 & 100 \\
Grade & 1 & 2 & 4.3 \\
& 2 & 16 & 34 \\
Total & 3 & 29 & 61.7 \\
& & 47 & 100 \\
\hline
\end{tabular}

Table 4. Total scales scores for test and re-test of Core, Core-Expanded and GYTS questionnaire GSHS Spearman's correlation $(\mathrm{N}=47)$.

\begin{tabular}{|lcc|}
\hline Module & Core P-Value & $\begin{array}{c}\text { Core-Expanded and } \\
\text { GYTS and Shisha } \\
\text { P-value }\end{array}$ \\
\hline Alcohol & 1 & 0.90 \\
Dietary Behaviors & 0.81 & 0.67 \\
Drug Use & 1 & 0.91 \\
Hygiene & 0.64 & 0.75 \\
Mental Health & 0.81 & 0.89 \\
Physical activity & 0.88 & 0.85 \\
Protective Factors & 0.93 & 0.90 \\
Sexual Behaviors & 0.99 & 0.79 \\
Tobacco & 0.95 & 0.88 \\
Violence & 0.92 & 0.78 \\
\hline
\end{tabular}




\begin{tabular}{lll|}
\hline Optional Module & 0.92 & 0.87 \\
(AIDS for Core and & & \\
Shisha for GYTS ) & & \\
\end{tabular}

\section{Administration of the GSHS and GYTS}

The self-report survey was administered to students in each school and its classrooms. One public health specialist and one community medicine specialist supervised the procedure; each student was first briefed on the survey. They were told that they could ask questions regarding the survey during administration of questionnaires. There were queries about the meaning of terms such as 'overdose' and 'amphetamine', for example. A 10-minute discussion followed the survey regarding the students' opinions and ideas about the survey and its clarity. On average, each student took around 100 minutes to complete the survey (Time 1).

Fourteen days later the re-test (Time 2) was administered. In Time 2, all students from each school again completed the survey with no follow-up discussions.

Students returned completed questionnaires to the researchers and were asked to wait for some minutes while their questionnaires were checked for important information (like their identification codes) which might be missing. Incomplete answer sheets were discarded in the data analysis.

Students who participated in the study received an identification code, which were used for labeling their related documents and their responses at test and re-test.

\section{Data analysis}

In order to assess the reliability and validity of Persian version of the GSHS questionnaires and GYTS, the internal consistency using Cronbach's alpha was estimated for all scales, items and the whole questionnaires. Test-retest reliability was assessed using Kendall's Tau coefficient of correlation at Time 1 (test) and Time 2 (re-test, with a 14-days interval). Spearman's correlation coefficient was calculated between total scale scores for test and re-test measures. For the validity of the questionnaires, Content Validity Index (CVI) and modified KAPPA, as a recent alternative in assessing the content validity were assessed based on expert perspectives. A minimum cumulative modified KAPPA score of 0.76 is expected to be achieved for content validation [8]. SPSS version 21 licensed to Mid Sweden University was used for analysis.

\section{Results}

The demographic characteristics of students who were administered the test and re-test are shown in Table 1. A majority of the respondents were 16-17 years of age and from third grade in High school (Table 1).

\section{Internal consistency of the Persian-version for Core, Core- Expanded GSHS and GYTS modules}

The internal consistency (Cronbach's $\alpha$ ) for Core-questionnaire varied considerably across modules (Table 2 ) ranged from 0.22 to 0.93 , and with total Cronbach's $\alpha$ of 0.69 for the whole questionnaire. The reliability of the questions was the lowest $(0,22)$ for physical activity and the highest $(0.93)$ for sexual behavior. Some items were excluded from modules due to the lack of making any scores in the internal reliability test. Each question has the different response option, for example, if the answer to item 1 (Have you ever had sexual intercourse?), while was "no," but the response to item 2 (How old were you when you had sexual intercourse for the first time?) was "I have never had sexual intercourse," the response was considered as inconsistent. The internal consistency for Core-Expanded and GYTS modules varied from 0.22 for Violence and unintentional injuries and 0.92 for Protective factors (Table 5). The Cronbach's $\alpha$ for the whole questionnaire was 0.90 , which is the good level of reliability.

\section{Test-retest reliability of the Persian-version for Core, Core- Expanded GSHS and GYTS}

The test-retest reliability of the Persian-version GSHS coremodule was assessed on 47 students from 20 randomly selected classes from 7 schools that had completed the questionnaire at Time 1 and Time 2. The distribution of scale scores for modules was similar at test and retest (Table 3) indicating good reliability of related subscales of the Persian version Core-module GSHS.

The test-retest reliability for the Core-Expanded GSHS and GYTS modules varied between 0.23 and 1 , with the lowest belonging to Dietary behaviors and the highest to the other items in modules.

\section{Total scales score for test and re-test}

Correlation between total scales scores for test and re-test was calculated based on Spearman's -correlation (Table 4). The highest score belonged to Alcohol and drug use (1.00), while the lowest to Hygiene (0.64). The Total scale score was calculated based on this formula: $100 \times$ (total scores of variables in scale) / Maximum ideal score.

Table 2. Internal consistency (Cronbach's alpha) of the core modules ( $N=47)$.

\begin{tabular}{|c|c|c|c|}
\hline Module & Item Number & Item Content & Cronbach's $\alpha$ \\
\hline \multirow[t]{6}{*}{ Alcohol Use } & & & 0.80 \\
\hline & 1 & How old were you when you had your first drink of alcohol? & \\
\hline & 2 & $\begin{array}{l}\text { During the past } 30 \text { days, on how many days did you have at least one drink } \\
\text { containing alcohol? }\end{array}$ & \\
\hline & 3 & $\begin{array}{l}\text { During the past } 30 \text { days, on the days you drank alcohol, how many drinks } \\
\text { did you usually drink per day? }\end{array}$ & \\
\hline & 4 & $\begin{array}{l}\text { During your life, how many times did you drink so much alcohol that you } \\
\text { were really drunk? }\end{array}$ & \\
\hline & 5 & $\begin{array}{l}\text { During your life, how many times have you got into trouble with your family } \\
\text { or friends, missed school, or got into fights, as a result of drinking alcohol? }\end{array}$ & \\
\hline \multirow{6}{*}{ Dietary Behaviors } & & & 0.25 \\
\hline & 1 & $\begin{array}{l}\text { During the past } 30 \text { days, how often did you go hungry because there was } \\
\text { not enough food in your home? }\end{array}$ & \\
\hline & 2 & During the past 30 days, how many times per day did you usually eat fruit? & \\
\hline & 3 & $\begin{array}{l}\text { During the past } 30 \text { days, how many times per day did you usually eat } \\
\text { vegetables? }\end{array}$ & \\
\hline & 4 & $\begin{array}{l}\text { During the past } 30 \text { days, how many times per day did you usually drink } \\
\text { carbonated soft drinks, such dough, Cola or delester? }\end{array}$ & \\
\hline & 5 & $\begin{array}{l}\text { During the past } 7 \text { days, on how many days did you eat food from a fast food } \\
\text { restaurant, such as hayda? }\end{array}$ & \\
\hline
\end{tabular}




\begin{tabular}{|c|c|c|c|}
\hline \multirow[t]{5}{*}{ Hygiene } & & & 0.45 \\
\hline & 1 & $\begin{array}{l}\text { During the past } 30 \text { days, how many times per day did you usually clean or } \\
\text { brush your teeth? }\end{array}$ & \\
\hline & 2 & During the past 30 days, how often did you wash your hands before eating? & \\
\hline & 3 & $\begin{array}{l}\text { During the past } 30 \text { days, how often did you wash your hands after using the } \\
\text { toilet or latrine? }\end{array}$ & \\
\hline & 4 & $\begin{array}{l}\text { During the past } 30 \text { days, how often did you use soap when washing your } \\
\text { hands? }\end{array}$ & \\
\hline \multirow[t]{7}{*}{ Mental Health } & & & 0.60 \\
\hline & 1 & During the past 12 months, how often have you felt lonely? & \\
\hline & 2 & $\begin{array}{l}\text { During the past } 12 \text { months, how often have you been so worried about } \\
\text { something that you could not sleep at night? }\end{array}$ & \\
\hline & 3 & $\begin{array}{l}\text { During the past } 12 \text { months, did you ever seriously consider attempting } \\
\text { suicide? }\end{array}$ & \\
\hline & 4 & $\begin{array}{l}\text { During the past } 12 \text { months, did you make a plan about how you would } \\
\text { attempt suicide? }\end{array}$ & \\
\hline & 5 & $\begin{array}{l}\text { During the past } 12 \text { months, how many times did you actually attempt } \\
\text { suicide? }\end{array}$ & \\
\hline & 6 & How many close friends do you have? & \\
\hline \multirow{5}{*}{ Physical Activity } & & & 0.22 \\
\hline & 1 & $\begin{array}{l}\text { During the past } 7 \text { days, on how many days were you physically active for a } \\
\text { total of at least } 60 \text { minutes per day? }\end{array}$ & \\
\hline & 2 & $\begin{array}{l}\text { During the past } 7 \text { days, on how many days did you walk or ride a bicycle to } \\
\text { or from school? }\end{array}$ & \\
\hline & 3 & $\begin{array}{l}\text { During this school year, on how many days did you go to physical education } \\
\text { (PE) class each week? }\end{array}$ & \\
\hline & 4 & $\begin{array}{l}\text { How much time do you spend during a typical or usual day sitting and } \\
\text { watching television, Playing computer games, talking with friends? }\end{array}$ & \\
\hline \multirow[t]{7}{*}{ Protective Factors } & & & 0.54 \\
\hline & 1 & $\begin{array}{l}\text { During the past } 30 \text { days, on how many days did you miss classes or school } \\
\text { without permission? }\end{array}$ & \\
\hline & 2 & $\begin{array}{l}\text { During the past } 30 \text { days, how often were most of the students in your school } \\
\text { kind and helpful? }\end{array}$ & \\
\hline & 3 & $\begin{array}{l}\text { During the past } 30 \text { days, how often did your parents or guardians check to } \\
\text { see if your homework was done? }\end{array}$ & \\
\hline & 4 & $\begin{array}{l}\text { During the past } 30 \text { days, how often did your parents or guardians } \\
\text { understand your problems and worries? }\end{array}$ & \\
\hline & 5 & $\begin{array}{l}\text { During the past } 30 \text { days, how often did your parents or guardians really } \\
\text { know what you were doing with your free time? }\end{array}$ & \\
\hline & 6 & $\begin{array}{l}\text { During the past } 30 \text { days, how often did your parents or guardians go through } \\
\text { your things without your approval? }\end{array}$ & \\
\hline \multirow[t]{6}{*}{ Sexual Behaviors } & & & 0.93 \\
\hline & 1 & Have you ever had sexual intercourse? & \\
\hline & 2 & How old were you when you had sexual intercourse for the first time? & \\
\hline & 3 & During your life, with how many people have you had sexual intercourse? & \\
\hline & 4 & $\begin{array}{l}\text { The last time you had sexual intercourse; did you or your partner use a } \\
\text { condom? }\end{array}$ & \\
\hline & 5 & $\begin{array}{l}\text { The last time you had sexual intercourse, did you or your partner use any } \\
\text { other method of birth control, such as withdrawal, rhythm (safe time), birth } \\
\text { control pills, or any other method to prevent pregnancy? }\end{array}$ & \\
\hline \multirow{6}{*}{ Tobacco Use } & & & 0.63 \\
\hline & 1 & How old were you when you first tried a cigarette? & \\
\hline & 2 & During the past 30 days, on how many days did you smoke cigarettes? & \\
\hline & 3 & $\begin{array}{l}\text { During the past } 30 \text { days, on how many days did you use any tobacco } \\
\text { products other than cigarettes, such as Shisha? }\end{array}$ & \\
\hline & 4 & During the past 12 months, have you ever tried to stop smoking cigarettes? & \\
\hline & 5 & $\begin{array}{l}\text { During the past } 7 \text { days, on how many days have people smoked in your } \\
\text { presence? }\end{array}$ & \\
\hline \multirow{5}{*}{$\begin{array}{l}\text { Violence and } \\
\text { Unintentional Injury }\end{array}$} & & & 0.45 \\
\hline & 1 & During the past 12 months, how many times were you physically attacked? & \\
\hline & 2 & During the past 12 months, how many times were you in a physical fight? & \\
\hline & 3 & During the past 12 months, how many times were you seriously injured? & \\
\hline & 4 & During the past 30 days, on how many days were you bullied? & \\
\hline \multirow[t]{4}{*}{ Optional Core Module } & & & 0.53 \\
\hline & $\begin{array}{l}1 \\
2\end{array}$ & $\begin{array}{l}\text { Have you ever heard of HIV infection or the disease called AIDS? } \\
\text { During this school year, were you taught in any of your classes about HIV }\end{array}$ & \\
\hline & 3 & $\begin{array}{l}\text { During this school year, were you taught in any of your classes how to avoid } \\
\text { HIV infection or AIDS? }\end{array}$ & \\
\hline & 4 & Have you ever talked about HIV infection or AIDS with your parents or & \\
\hline
\end{tabular}




\section{Total $\alpha$ for 48 Items \\ guardians? \\ Content validity index and modified Kappa validity of GSHS Core, Core-Expanded and GYTS were rated by five community medicine experts. They were asked to mark the content of translated questionnaires as A) Highly relevant, B) Quite relevant, C) Somewhat relevant, and D) Not relevant. Both CVI and modified scores were calculated following this procedure: If 4 out of 5 respondents marked 'A', the $C V I=0.8$ and Modified $=0.763$; if all of them chose 'A' both CVI and modified $=1$; and if 3 out of 4 respondents marked ' $A$ ', the $C V I=0.75$ and modified= 0.66 (Table 6).}

\section{Discussion}

The Global School Based Student Health Survey (GSHS) was designed to assess the behavioral risk factors and protective factors in the main areas of morbidity and mortality among students in different countries. Testing the reliability and validity of translated questionnaires is a neglected area. Despite as mentioned before many countries have used the translated questionnaires with out testing its reliablity and validity.In Iran the GYTS questionnaires were used 2 times for data collection without testing the validity and reliability of these questionnaires [7]. Validity and reliability are the minimum prerequisites for acceptance of a translated questionnaire, besides the accuracy of its translation [9]. This is the first study in Iran that tested the reliability and validity of all Core, Core-Expanded (version 2009) and GYTS (version 2012), and Optional module for Shisha smoking Persian version questionnaires.

The data from this study support the acceptable reliability and validity of the majority of the translated questions adapted from GSHS Core and Core- Expanded and GYTS content for Iranian students. The number of modules with unacceptable reliability was small. These modules related to the internal consistency of dietary behaviors, physical activity and violence in both Core and Core-Expanded questionnaires, these items which have shown lower reliability in comparison with other items, suggesting caution in interpretation of data regarding those items. May be reliability of these items affected by variation in stability of knowledge, health behaviors and attitudes over time of interval assessed and the number of sample size, number of questions and their different response options.

This study considered all of the items in the reliability tests, and it is one of the strength and at the same time one of the main weak points of this study, unlike other studies has reported the reliability of only Core questionnaire and small scales of items, and has deleted the items that reduce their consistency level. The total Cronbach's a coefficient, for the whole Core, CoreExpanded and GYTS questionnaires showed an acceptable reliability of the questionnaires. Cronbach's alpha for these questionnaires were 0.69 for Core and 0.90 for Core Expanded and GYTS indicating good internal consistency for the items of these questionnaires. The stability of responses was estimated over a 14-day interval which is followed in the test (Time1) and retest (Time2) approach. Similar score distributions on tests taken with 14 days of interval supported the stability (Table 3 ) of these scales, indicating acceptable reliability of the Persian version of GSHS Core, Core Expanded and GYTS questionnaires. Content validity index and modified Kappa validity have shown the acceptable validity of modules in all questionnaires.

Table 3. Test-retest reliability of the Persian-version GSHS $(N=47)$.

\begin{tabular}{|c|c|c|c|c|c|}
\hline Module & Item Number & Kendall's tau_b & Module & Item Number & Kendall's tau_b \\
\hline \multirow[t]{2}{*}{ Alcohol } & & & Protective Factors & & \\
\hline & All 6 items & 1 & & 1 & 0.56 \\
\hline \multirow[t]{8}{*}{ Dietary Behaviors } & & & & 2 & 0.68 \\
\hline & 1 & 0.99 & & 3 & 0.73 \\
\hline & 2 & 0.93 & & 4 & 0.85 \\
\hline & 3 & 0.80 & & 5 & 0.82 \\
\hline & 4 & 0.72 & & 6 & 0.91 \\
\hline & 5 & 0.67 & Sexual Behavior & & \\
\hline & 6 & 0.71 & & $1,2,3,4$ & 1 \\
\hline & 7 & 0.79 & & 5 & 0.95 \\
\hline Drug Use & All 4 items & 1 & Tobacco & & \\
\hline \multirow[t]{5}{*}{ Hygiene } & & & & 1 & 0.99 \\
\hline & 1 & 0.63 & & 2 & 1 \\
\hline & 2 & 0.61 & & 3 & 0.91 \\
\hline & 3 & 0.51 & & 4 & 0.95 \\
\hline & 4 & 0.46 & & 5 & 0.79 \\
\hline \multirow[t]{7}{*}{ Mental Health } & & & & 6 & 1 \\
\hline & 1 & 0.74 & $\begin{array}{l}\text { Violence and } \\
\text { Unintentional Injury }\end{array}$ & & \\
\hline & 2 & 0.82 & & 1 & 0.69 \\
\hline & 3 & 1 & & 2 & 0.99 \\
\hline & 4 & 0.76 & & 3 & 0.98 \\
\hline & 5 & 1 & & 4 & 0.93 \\
\hline & 6 & 0.59 & & 5 & 1 \\
\hline \multirow[t]{7}{*}{ Physical Activity } & & & & 6 & 0.89 \\
\hline & 1 & 0.73 & & 7 & 0.84 \\
\hline & 2 & 0.79 & $\begin{array}{l}\text { Optional Core } \\
\text { Module }\end{array}$ & & \\
\hline & 3 & 0.78 & & 1 & 0.84 \\
\hline & 4 & 0.81 & & 2 & 0.83 \\
\hline & & & & 3 & 0.95 \\
\hline & & & & 4 & 1 \\
\hline
\end{tabular}


Iran is an Islamic country with diverse traditional habits, culture and behaviors. Today Iran is in transitional stage from a traditional to a modern society, and so cultural adaptation has an important role in developing any measure instrument.

Some modules such as alcohol consumption and drug use are considered illegal in the Iranian society, and sexual behaviors are considered illegal until getting marriage, However, risky behaviors from juveniles may result in addiction to alcohol and drug as well as sexually transmitted diseases and unwanted pregnancies, so mere being illegal does not mean that there is no incidence of these behaviors.

The present study for the first time has used candid questions regarding modules that are regarded as stigma. This study shows the Persian version of GSHS and GYTS is reliable and valid and can be used in Persian speaking communities.
Funding: This work was supported by the Research Committee of Tabriz University of Medical Sciences under Grant number $5 / 4 / 8860$.

Ethical approval: Ethics Committee of Tabriz University of Medical Sciences.

Conflict of interests: The authors declare no conflict of interest.

Acknowledgement: The authors wish to thank the study participants for their contribution to the study, as well as study investigators and staff. The authors would specifically like to thank Dr. Vahid Nimechi Salem, Mr. Abolfazl Jeddi, Ms. Akram Allahyari, Dr. Mahasti Alizadeh, Dr. Rana Hosseini, Dr. Siavash Moradi, Dr. Elham Khanlarzadeh, Dr. Parvin Delpasand, Dr. Akram Beheshti, and Ms. Ghasemiyeh for their research and administrative assistance.

Table 5. Internal consistency (Cronbach's alpha) of the Core-Expanded and GYTS modules $(N=47)$.

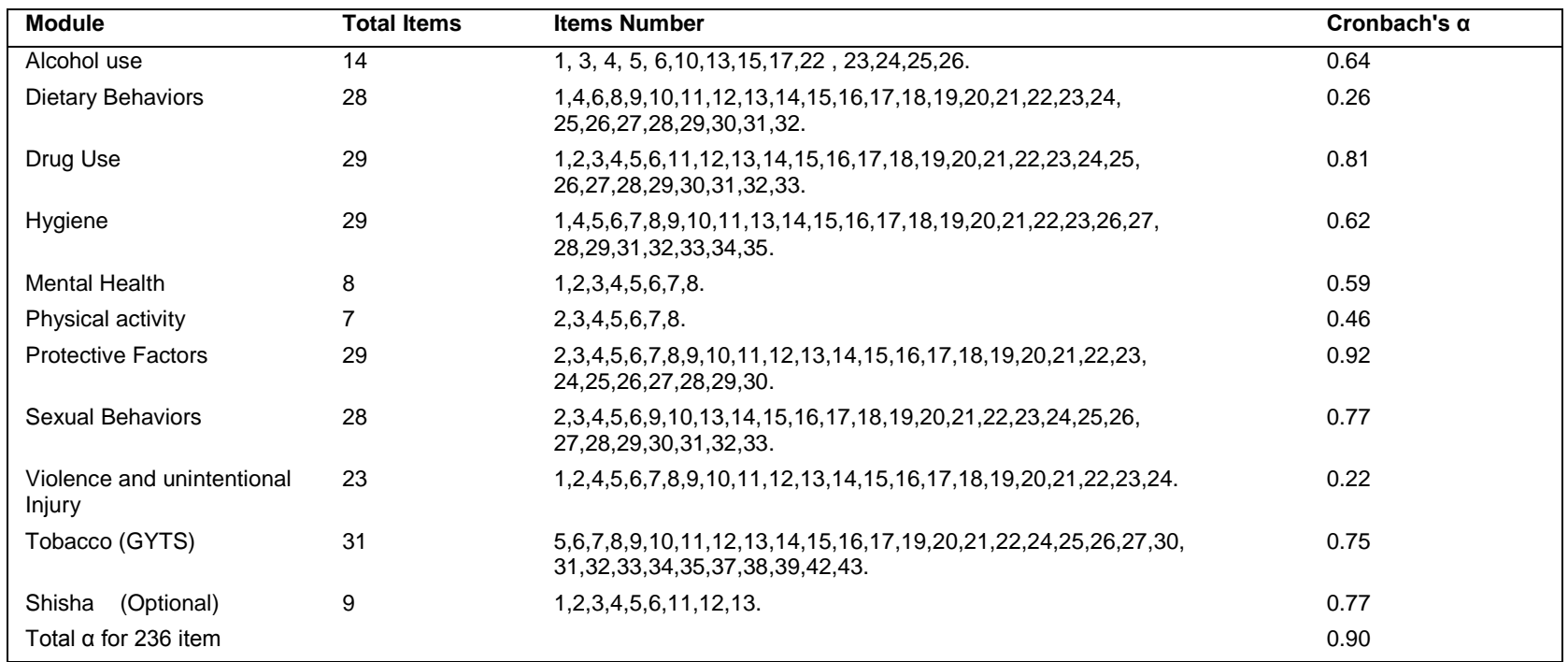

Table 6. Content validity index and modified Kappa validity.

\begin{tabular}{|c|c|c|c|c|}
\hline Module & CVI (Core) & Modified (Core) & CVI (Core-Expanded and GYTS) & Modified \\
\hline Alcohol & 1 & 1 & 0.91 & 0.89 \\
\hline Dietary behaviors & 1 & 1 & 1 & 1 \\
\hline Drug & 1 & 1 & 0.98 & 0.98 \\
\hline Hygiene & 1 & 1 & 0.99 & 0.99 \\
\hline Mental Health & 1 & 1 & 0.97 & 0.97 \\
\hline Physical activity & 1 & 1 & 1 & 1 \\
\hline Protective factors & 1 & 1 & 1 & 1 \\
\hline Sexual behaviors & 1 & 1 & 0.93 & 0.92 \\
\hline Tobacco & 1 & 1 & 1 & 1 \\
\hline $\begin{array}{l}\text { Violence and unintentional } \\
\text { injury }\end{array}$ & 1 & 1 & 0.95 & 0.95 \\
\hline $\begin{array}{l}\text { Optional module } \\
\text { (AIDS for Core and Shisha } \\
\text { for GYTS ) }\end{array}$ & 1 & 1 & 1 & 1 \\
\hline Total & 1 & 1 & 0.97 & 0.97 \\
\hline
\end{tabular}

\section{References}


1. WHO: Youth violence. Fact sheet N.356. August 2011.[http://www.who.int/mediacentre/factsheets/fs3 56/en/]

2. Blum RW, Nelson-Mmari K: The health of young people in a global context. $J$ Adolesc Health 2004,35(5):402-18.

https://dx.doi.org/10.1016/j.jadohealth.2003.10.007.

3. Becker $A E$, Roberts $A L$, Perloe A, Bainivualiku A, Richards LK, Gilman SE, et al: Youth health-risk behavior assessment in Fiji: the reliability of Global School-based Student Health Survey content adapted for ethnic Fijian girls. Ethn Health 2010,15(2):181-97. doi: https://dx.doi.org/10.1080/13557851003615552.

4. Reza A, Mercy JA, Krug E: Epidemiology of violent deaths in the world. Inj Prev 2001,7(2):104-11. doi: https://dx.doi.org/10.1136/ip.7.2.104

5. WHO: Global School-based Student Health Survey (GSHS). [http://www.who.int/chp/gshs/en/].
6. Chen PL, Chiou HY, Chen YH: Chinese version of the Global Youth Tobacco Survey: cross-cultural instrument adaptation. BMC public health 2008,8:144. doi: https://dx.doi.org/10.1186/1471-2458-8-144.

7. WHO: Global youth tobacco survey. Country facts sheets. 2010.

[http://www.emro.who.int/images/stories/tti/documents/ GYTS FS IRI R1.pdf http://www.emro.who.int/images/stories/tfi/documents/G YTS_FS_IRI_R2.pdf]

8. Polit DF, Beck CT, Owen SV: Is the CVI an acceptable indicator of content validity? Appraisal and recommendations. Res Nurs Health 2007,30(4):459-67. Doi: https://dx.doi.org/10.1002/nur.20199.

9. Streiner DL, Norman GR: Basic Concepts in Health Measurement Scales: A Practical Guide to their Development and Use. $3^{\text {rd }}$ ed. New York, NY: Oxford University Press; 2003: 4-13. 